\title{
Digitale Medien als Therapiebühne und Intermediärobjekte in der Therapie von traumatisierten Kindern und Jugendlichen
}

\section{Einsatz von Smartphone und Co. als Therapietools mit Kindern und Jugendlichen, Fallvignetten aus der psychotherapeutischen Praxis}

\author{
Ulrike Altendorfer-Kling \\ Online publiziert: 23. September 2021 \\ (C) Springer Fachmedien Wiesbaden GmbH, ein Teil von Springer Nature 2021
}

Zusammenfassung Dieser Beitrag der Zeitschrift für Psychodrama und Soziometrie setzt sich mit der Frage auseinander, wie wir digitale Medien in der Psychodramatherapie mit Kindern und Jugendlichen wirkungsvoll zum Einsatz bringen können. In Krisenzeiten ist es für uns Menschen besonders wichtig, den Kontakt zueinander aufrechterhalten, einander unterstützen und voneinander lernen zu können. In diesem Beitrag wird ein flexibler Umgang mit den neuen technischen Möglichkeiten gezeigt, die zur Erweiterung der Therapiemethode Psychodrama führen und besonders in der Traumatherapie hilfreich sind.

Schlüsselwörter Digitale Medien · Psychodrama - Instant Messenger · Videotelefonie · Intermediärobjekte · Covid-19 · Traumatherapie · Krisenintervention · Videokonferenztechnik

Dr. Ulrike Altendorfer-Kling $(\bowtie)$

Praxis für Psychodramapsychotherapie, Mönchsberg 33, 5020 Salzburg, Österreich

E-Mail: praxis@draltendorfer.at 


\title{
Digital media as therapeutic stage and intermediary objects in the psychotherapy of traumatized children and adolescents
}

Use of smartphones and Co. as therapeutic tools with children and adolescents, case vignettes from psychotherapeutic practice

\begin{abstract}
This contribution in the journal Zeitschrift für Psychodrama und Soziometrie deals with the question of how we can use digital media effectively in Psychodramatherapy with children and adolescents. In times of crisis it is most important for us human beings to keep in contact, support each other and learn from each other. This contribution presents the flexible use of the new technical options, which lead to expansion of Psychodrama psychotherapy and which are useful especially in Traumatherapy.
\end{abstract}

Keywords Digital Media · Psychodrama - Instant Messaging · Video telephony · Intermediate Objects · Covid-19 · Traumatherapy $\cdot$ Crisis Intervention

\section{Einleitung}

Digitale Medien helfen bei der Kommunikation mit sich selbst und anderen und werden in der psychotherapeutischen Arbeit mit traumatisierten Kindern und Jugendlichen erfolgreich eingesetzt.

Viele von uns fragen sich, ob und wie mit digitalen Medien der Begegnungsmoment nach Moreno gelingen kann. Unter anderem ist Agnes Blahacek (2020) der Frage nachgegangen, ob über Begegnung via Bildschirm denn überhaupt die Wahrscheinlichkeit einer ,unvergesslichen Erfahrung völliger Gegenseitigkeit“ besteht, wie es Moreno formulierte (1959, zit. nach Hutter und Schwehm 2009).

Jacob Levi Moreno hat uns die Antwort auf diese Frage mit seinem Film „Psychodrama in Action“ gegeben (Moreno, Video 2020). Er ist der Meinung, dass Psychodrama auch mithilfe fernsehtechnischer Mittel auf Distanz wirksam ist, und dass die Menschen auch durch ausschließliches Beobachten im Sinne der Observationskatharsis Heilung finden. Er führte bereits im Mai 1964, einmal wöchentlich im Camarillo State Hospital, California, PatientInnen einer geschlossenen Abteilung einen Film vor, in dem er eine Psychodramatherapie-Sitzung mit PatientInnen der offenen Station leitete. Damit wollte Moreno Psychodrama als „Massentherapiemethode" etablieren und für alle Menschen, auch für die mit geringen finanziellen Ressourcen, erreichbar machen. Er eröffnete eine „Road to the Future ...“. Insofern ist die Digitalisierung der Methode Psychodrama eine Weiterentwicklung seiner Vision, die mithilfe der heutigen technischen Möglichkeiten, eine weitere Umsetzung seiner Ideen ermöglicht.

Morenos Begegnungsbegriff ist laut Agnes Blahacek (2020) natürlich viel weitreichender zu definieren als über das rein physische Aufeinandertreffen von Menschen und umfasst mehrere Dimensionen. Dem Begegnungskonzept kommt in der täglichen psychodramatherapeutischen Arbeit eine grundlegende Bedeutung zu. Das gilt auch für die Begegnung mit Kindern und Jugendlichen. Mit den digitalen Medien eröffnen sich neue Formen der Begegnung und der Traumaaufarbeitung in der 
Therapie. Dafür brauchen wir einen flexiblen Umgang mit den neuen technischen Möglichkeiten, die zur Erweiterung der Möglichkeiten der Therapiemethode führen.

Die digitale Bühne ist für Kinder und Jugendliche etwa ab dem Alter von vier bis fünf Jahren gut einsetzbar. Sobald das Kind einen Rollentausch und Rollenwechsel durchführen kann, ist es prinzipiell möglich, Handys und andere digitale Medien als Intermediärobjekte oder Therapieunterstützer einzusetzen. Sinnvoll finde ich den Einsatz digitaler Medien bei Kindern im Vorschulalter nur, wenn diese Medien schon zur alltäglichen Erfahrungswelt des Kindes gehören, das Kind entsprechend damit umgehen kann und sie in der Therapie als Vermittler für die Kommunikation mit sich selbst oder anderen eingesetzt werden, wie es Pruckner (2004) beschreibt.

Kritisch sehe ich es, wenn in der Symbolarbeit, vermittelt durch digitale Medien oder auch im Präsenzmodus, der Schutz durch das Symbol durch eine Erklärung oder Deutung durch den/die TherapeutIn im Spiel oder danach aufgehoben wird.

Im psychodramatischen Spiel und allgemein in der Psychodramatherapie wird von TherapeutInnen oft sehr viel Geduld gefordert, sie müssen sich dem innerseelischen Tempo des Kindes oder Jugendlichen anpassen. Manchmal braucht die gemeinsame Arbeit sorgfältiges und genaues Herausarbeiten von Details oder auch mehrfache Wiederholung von derselben Szene, um zu besserem Verständnis und Lösungsansätzen zu kommen. Kinder und Jugendliche zeigen ihr Tempo sehr genau vor. In der Arbeit mit PatientInnen mit Traumafolgestörungen ist es besonders wichtig, dieses innerseelische Tempo zu respektieren anstatt vorschnelle Interventionen zu setzen, besonders, wenn es zu einem stockenden Spielverlauf oder zum Spielabbruch kommt, da es sonst zur Retraumatisierung kommen kann.

Das Spiel eines traumatisierten Kindes kann über Wochen oder Monate erschreckende, gewaltsame und sexuelle Inhalte ausspielen, in den aggressiven Mustern der Täter-Opfer-Umkehr verharren, ohne dass die „heilsame Kraft“ des Spiels sichtbar wird, ohne dass es zu einer Verarbeitung und zu einer sichtbaren Persönlichkeitsentwicklung kommt. Es besteht die Gefahr, dass das Spiel die Als-Ob-Qualität verliert, Kind und TherapeutIn zu TäterIn und Opfer werden und damit die Spielbühne auf die Begegnungsbühne rutscht (Pruckner 2002). Aichinger (2012) sieht im Rollenspiel eine große Chance, dass Kinder ihre traumatischen Erfahrungen geschützt darstellen, verarbeiten und mit allen Sinnen eine Neuerfahrung machen können. Pruckner (2002) arbeitet ausschließlich mit Intermediärobjekten, weil die Rolle, die der/die TherapeutIn übertragen bekommt, gerade bei traumatisierten Kindern oft schwer zu spielen ist.

Die Täterintrojektion dient als Schutzvorgang, der vor überwältigender Ohnmacht schützt und sie ermöglicht einen Rest Einfluss und Kontrolle über das Verhalten des/r TäterIn, was in manchen Situationen lebensnotwendig sein kann (Sachsse 2004). Die entsprechenden Rollen können auf der digitalen Bühne ebenso gespielt werden wie auf der analogen Bühne. In der digitalen Traumatherapie gelten dieselben Regeln wie auf der Psychodramabühne im realen Raum.

Wir nützen die Surplus Reality, die wie von einigen Jugendlichen rückgemeldet, in der digitalen Therapie mehr Distanzierungsmöglichkeiten und zugleich Orientierung und Halt über den klaren Rahmen des technischen Geräts bietet. 


\section{Digitaler Einsatz zentraler Techniken wie Doppeln, Spiegeln, Rollenwechsel und Rollentausch, Rollenspiel, Sharing, Soziometrie}

Digitale Medien können in der Psychodrama-Therapie vielfach eingesetzt werden. Eine Möglichkeit ist, sie als Vermittler im Sinne von Intermediärobjekten zu verwenden. Der Einsatz von Intermediärobjekten in der Kinderpsychodramatherapie ist wie Rojas-Bermúdez (1985) und auch Pruckner (2001) bemerken, immer als Vermittler in einer sonst unmöglichen Kommunikation $\mathrm{zu}$ verstehen, ähnlich einem Satelliten, der als Vermittler für ein Telefongespräch fungiert, das direkt unmöglich wäre. Das Intermediärobjekt in diesem Sinne ist ein reales Objekt, das eine neue Funktion als Vermittler, innerhalb einer unterbrochenen oder bisher nicht vorhandenen Kommunikation, erhält. In der psychotherapeutischen Arbeit mit Kindern und Jugendlichen zeigt sich immer wieder die Bedeutung der digitalen Medien als Intermediärobjekte (Pruckner 2004, S. 276), die im Sinne einer unsichtbaren Nabelschnur mit den Bezugspersonen Verbundenheit schaffen. Die Kinder und Jugendlichen wählen ihr subjektiv passendes Intermediärobjekt aus (Abb. 1).

Ein Intraintermediärobjekt kann nach Biegler-Vitek und Wicher (2017, S. 272) neben der vermittelnden Funktion von Intermediärobjekten auch eine unterstützende oder kreative Funktion haben. Es fördert die Kommunikation mit anderen und mit sich selbst. Die Auswahl erfolgt hier durch den/die PatientIn.

\subsection{Doppeln}

Die digitalen Medien sind durch ihre vermittelnde Funktion ebenfalls für einfühlendes und stützendes Doppeln nutzbar. Wenn ich in einer Videotelefoniesitzung dopple, verwende ich für mich als Therapeutin ein Symbol und bitte den/die PatientIn, es für mich auf der Tischbühne zur Figur des/der PatientIn dazuzulegen. Danach wird

\begin{tabular}{|c|c|c|c|}
\hline & Intermediärobjekt & Intraintermediärobjekt \\
\hline & & Interpersonale Kommunikation. & $\begin{array}{l}\text { Intrapersonale und interpersonale } \\
\text { Kommunikation. }\end{array}$ \\
\hline \begin{tabular}{|l|} 
Unterstützende \\
Funktion
\end{tabular} & $\begin{array}{l}\text { Das Objekt unterstützt } \\
\text { oder hebt etwas hervor, } \\
\text { das bereits passiert. }\end{array}$ & \multicolumn{2}{|c|}{$\begin{array}{c}\text { - Aufwärmphase: } \\
\text { Hilfsmittel für die Definition } \\
\text { der Rollen und Situationen. } \\
\text { - Spielphase: } \\
\text { Hilfssmittel zur Unterstützung } \\
\text { der Inszenierung. }\end{array}$} \\
\hline $\begin{array}{l}\text { Vermittelnde } \\
\text { Funktion }\end{array}$ & $\begin{array}{l}\text { Die Präsenz des Objekts } \\
\text { ist determinierend für } \\
\text { Ausdruck und Kommu- } \\
\text { nikation. }\end{array}$ & $\begin{array}{l}\text { Das Objekt wird vom Leiter } \\
\text { eingesetzt, um die abgebrochene } \\
\text { Kommunikation herzustellen. } \\
\text { Der Klient kommuniziert mit } \\
\text { dem Objekt, nicht mit der Person, } \\
\text { die es einsetzt. }\end{array}$ & $\begin{array}{l}\text { Das Objekt wird vom Klienten eingesetzt. } \\
\text { Es ist primär Katalysator in der Kommuni- } \\
\text { kation mit sich selbst, hilft sekundär in der } \\
\text { Kommunikation mit anderen. }\end{array}$ \\
\hline $\begin{array}{l}\text { Kreative } \\
\text { Funktion }\end{array}$ & $\begin{array}{l}\text { Das Objekt wird vom } \\
\text { Klienten produziert. } \\
\text { Der Sinninhalt repräsentiert } \\
\text { unbekannte und abgelehnte } \\
\text { Inhalte und wird während } \\
\text { der Erschaffung sichtbar. }\end{array}$ & & $\begin{array}{l}\text { - Produkte ästhetischer Kommunikations- } \\
\text { techniken: Zeichnungen, Collagen, Texte, } \\
\text { selbstgestaltete Videofilme etc. } \\
\text { - Prozess der Gestaltung. } \\
\text { - Inszenierung mit dem Objekt }\end{array}$ \\
\hline
\end{tabular}

Abb. 1 Graphik nach J. Rojas-Bermúdez (übersetzt und gestaltet von M. J. Ancochea Serraima) (Pruckner 2004, S. 276) 
es wieder an den Bühnenrand gelegt. Dadurch wird Rollenklarheit auch via digitale Medien lebbar.

Die Tischbühne kann im Zimmer des/der PatientIn sein. Dann muss ich darauf deuten, wenn ich doppeln will. Der Vorteil dabei ist, dass der/die ProtagonistIn die Figuren und Symbole selbst bewegt. Wenn die Tischbühne in meinem Praxisraum liegt, dann bewege ich als Therapeutin die Figuren und frage viel nach. Das ist von Vorteil, wenn ich mit sehr gehemmten Menschen arbeite, die mich noch verstärkt als Hilfs-Ich brauchen.

Fallbeispiel: Ein 17-jähriger Jugendlicher, der auf der Tischbühne in seiner Wohnung während einer Videotelefoniesitzung mit Symbolen eine Szene mit seinem Vater spielte, in der dieser ihn schlug und schimpfte, er selbst sich und seinen Kopf mit den Armen schützend duckte, war von seiner fehlenden Wehrhaftigkeit in dieser Situation enttäuscht. Er spürte zwar Wut und Ärger gegen den Vater aufsteigen, konnte dies aber nicht ausdrücken, weil er sich diesem unterlegen fühlte. Zunächst hatte er auch im Spiel keinen Zugang zu diesen verbotenen Gefühlen. In der realen Situation war das möglicherweise der beste Weg mit der Gewalt durch den Vater umzugehen, da dieser bei gezeigter Aggression erfahrungsgemäß noch härter zuschlug.

Auf der Tischbühne wurde es jedoch möglich, dass ich ihm meine Figur zur Seite stellen ließ. Der Patient legte den Finger auf die Figur und ich doppelte seine möglichen Gefühle. Als ich fertiggesprochen hatte, legte der junge Mann die Figur, die mich symbolisierte, an den Bühnenrand.

Er berührte die eigene Figur und sprach aus, was er empfand, er bestätigte manches und wehrte bei anderen Gefühlen ab. Die Traurigkeit und die Scham zum Beispiel empfände er nicht, aber die Wut werde immer stärker und differenzierter. Es entwickelte sich der Ausdruck für eine Wut, die sich zunächst gegen den Patienten selbst und sein Versagen richtete und dann eine weitere Wut, die sich gegen den Vater richtete, der ihn so misshandelte.

Gemeinsam entwickelten wir die Szene weiter, hin zu verschiedenen Ausdrucksformen der Wut und des Selbstschutzes. Was wollte der Patient in der Surplus Reality des Spieles nun dem Vater sagen? Wie wollte er sich wehren? Welche Hilfsmittel würde er dazu benötigen? Wie konnte er sich selbst vor weiterer Gewalt schützen? Für den Patienten entstand im Spiel eine gute Lösung, als er die Idee hatte, „Kingsman" würde plötzlich auftauchen und sich zwischen ihn und den Vater stellen. In den folgenden Videositzungen beschäftigten wir uns mit „Kingsman“ und dessen Fähigkeiten. Wir kreierten einen individuell gestalteten „Kingsman“, der die Durchsetzungsfähigkeit und Intelligenz besaß, die meinem Patienten so wichtig war, um in Konflikt- und Angstsituationen bestehen zu können.

In einer weiteren Videotelefoniesitzung wurde ein Rollentausch mit der Figur des „Kingsman“ angeleitet, der den Menschen, die es in der Vergangenheit nicht gut mit meinem Patienten gemeint hatten, ordentlich die Meinung sagte und ihnen klarmachte, was sie bisher übersehen und falsch gemacht haben.

Die Tischbühne befand sich im Raum des Patienten, daher konnte er selbst leicht von seiner Ich-Rolle in die des „Kingsman“ wechseln und den symbolisierten Mit- 
menschen sein Plädoyer halten. „Kingsman“ wurde zum vermittelnden Intraintermediärobjekt für den jungen Mann, das ihm durch seine kreative Funktion zu mehr Durchsetzungskraft im Leben verhalf. Ein Rollentausch mit der Figur des Vaters war zu diesem Zeitpunkt noch nicht möglich.

\subsection{Spiegeln}

Die Spiegeltechnik als zentrale Psychodrama Technik kann ebenfalls mit digitalen Medien Einsatz finden. Diese ermöglichen ein Spiel mit Nähe und Distanz auf ihre eigene Weise, und die Spiegeltechnik des Psychodramas kann durch Videotelefonie beispielsweise mithilfe von Screenshots umgesetzt werden, die an die PatientInnen geschickt werden. Auch per SMS- oder Messenger-Systemen wird die Spiegeltechnik sehr erfolgreich eingesetzt.

Fallbeispiel: Erfolgreiches digitales Spiegeln gelang in der Traumatherapie einer jugendlichen Patientin aus meiner Praxis. Sie war davon überzeugt, aufgrund ihrer traumatisierenden Lebenserfahrungen so „,beziehungsgestört zu sein“, wie sie sagte, dass sie sich weder liebevoll gegenüber ihrer Katze, geschweige denn irgendeinem geliebten Menschen verhalten könnte.

Sie brachte dieses Thema immer wieder in verschiedenen Versionen in die Therapiesitzungen im Präsenzmodus ein, und wir suchten gemeinsam nach Situationen, in denen ihr liebevolle Begegnungen und Beziehungsgestaltung gelangen. Oft musste ich auch einfach ihre Erzählungen zur Kenntnis nehmen und konnte ihr keine Spiegelung aus ihrem Alltagsleben geben. Die gelungenen Begegnungen zwischen uns und Erlebnisse von Nähe innerhalb der Therapiesituation wertete sie regelmäßig ab als im geschützten Rahmen erlebte, im Alltag nicht umsetzbare Momente.

Als nun im Frühjahr 2020 die Therapie ausschließlich digital per Videotelefonie weitergeführt werden konnte, bekam ich Einblick in ihre Wohnsituation. Sie machte es sich immer wieder zu Hause bequem und richtete sich für die Therapiesitzung gemütlich ein. Eines Morgens kam es dazu, dass die Katze der Patientin im Hintergrund durch das Bild striff, zunächst in einiger Entfernung, dann bereits unmittelbar hinter ihr auf der Sofalehne und schließlich setze sie sich schnurrend auf den Schoß der Patientin, die mit mir sprach. Für mich hatte es den Anschein, als wollte die Katze die Aufmerksamkeit der jungen Frau auf sich ziehen. Sie krallte sich in ihre Kleidung, suchte immer wieder ihren Blickkontakt und bot sich zum Austausch von Zärtlichkeiten an. Die Katzenbesitzerin schien sich darüber zu freuen, streichelte das Tier und es kam wiederholt zu liebevollem Blickkontakt und Austausch von Zärtlichkeiten zwischen den beiden. Das Gespräch zwischen uns beiden wurde ungehindert weitergeführt. Ich bot meiner jungen Patientin an, Screenshots zu machen und sie ihr zu schicken. Sie freute sich sehr über diese spontanen Beweise ihrer Kompetenzen und die Erinnerung an diese schönen Momente und lächelte zufrieden in die Kamera. Sharings aus dem Zusammenleben mit Katzen von meiner Seite rundeten die Sitzung ab und ermöglichten eine lustvolle und spielerische Auseinandersetzung mit dem Thema Beziehungsgestaltung. 
Abb. 2 Screenshot von Beziehungsszene Mensch-Katze; Altendorfer-Kling (2020)

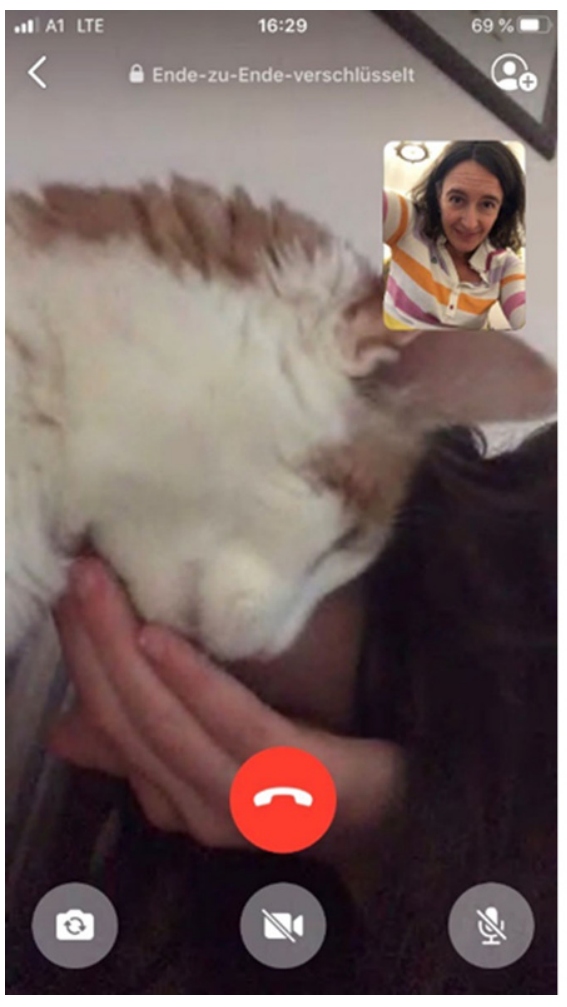

Erfreulicherweise berichtete die Patientin zwei Monate später von dem erstmaligen Versuch, seit mehreren Jahren, wieder eine Liebesbeziehung einzugehen. Sie hatte mit der Person schon seit sechs Monaten Mailkontakt und nun hatte sie den nächsten Schritt gewagt, ein persönliches Treffen der beiden wahrzunehmen. Hier habe ich die unterstützende Funktion des Intermediärobjekts ,Screenshot“ für etwas eingesetzt, was bereits passierte, die gelingende Beziehungsgestaltung.

Per Videotelefonie erhält der/die TherapeutIn andere Eindrücke von der Situation und der unmittelbaren Umgebung der PatientInnen in ihrem privaten Umfeld, es können bestimmte Dinge oder das eigene Haustier gezeigt oder versteckt werden, während der/die PatientIn sich frei und unmittelbar in seinem/ihrem Umfeld bewegt. So ist eine andere Form der Begegnung möglich, obwohl meist einige Kilometer in der Realität zwischen PatientIn und TherapeutIn liegen (siehe Abb. 2).

Das folgende Buch und ein Spielfilm sind weitere sehr gut in der Psychodramatherapie einsetzbare Medien, die mithilfe digitaler Hilfsmittel entstanden sind: Im Buch „Seelenleben“" von Schulte-Markwort (2020) geben 20 Jugendliche Einblick in ihre Psyche, und ihre persönlichen Berichte werden durch Briefe ihres Therapeuten an sie beantwortet. Die psychodramatische Spiegelung der Jugendlichen wird durch eine Spiegelung über digitale Medien ergänzt, indem im Rahmen eines Fotoshootings sehr persönliche Fotos der jugendlichen Menschen entstanden sind. Hier wurde vom Autor die Beobachtung aufgegriffen, dass Selfies eine wichtige Ausdrucksform von 
Jugendlichkeit geworden sind. Im Rahmen des Projekts „Non-Selfies“ wurde an der Klinik für Kinder- und Jugendpsychiatrie des Universitätsklinikums Hamburg-Eppendorf zusammen mit Interviews aus der kinder- und jugendpsychiatrischen Praxis die psychodramatische Spiegeltechnik mit allen zur Verfügung stehenden Medien umgesetzt. Das Buch zeigt sehr einfühlsam eine gelungene therapeutische Intervention, ist aber auch im Rahmen der Psychoedukation und Entstigmatisierung von Menschen mit psychischen Erkrankungen sehr gut einsetzbar, weil es die Empathie der LeserInnenschaft für die heutigen Jugendlichen und ihre Situation fördert.

Als Intermediärobjekt kann es in der Therapie eingesetzt werden, indem der/die PatientIn es in Händen hält, mit dem Buch zunächst in Dialog geht und einen Rollentausch vollzieht. Was erzählt das Buch ,Seelenleben“ über die Person? Wie geht es ihr, welche Fotos findet sie? Wo im Buch kommt die Person vor?

Der Animationsfilm „Alles steht Kopf“ (2015) ermöglicht ebenfalls sehr gute Einblicke in das Seelenleben von Kindern und Jugendlichen und ist in der Psychodrama-Therapie ideal als Therapieunterstützer und Anschauungsmaterial einsetzbar, um die Methode und Techniken auf der inneren und äußeren Bühne den Kindern, Jugendlichen und ihren Eltern näher zu bringen.

\subsection{Rollenwechsel}

\subsubsection{Rollenwechsel im Einzelsetting}

Fallbeispiel: Ein zehnjähriger Bub, der bei seiner alleinerziehenden Mutter lebt, hat in seiner Psychodramatherapie im Präsenzmodus sein Trauma betreffend der elterlichen Streitigkeiten und der Trennung der Eltern, die ihn verstummen und ihn Mobbingerfahrungen in der Schule ohne Gegenwehr oder hilfesuchendes Verhalten erdulden ließen, in einem selbstgedrehten Stop-Motion-Film am Handy dargestellt. So war es möglich, in der Spielphase der Sitzungen ein Tierfigurenspiel mit ihm zu gestalten, in dem er selbst als Bärenkind, seine Wut auf die Eltern als Krokodil und seine Mutter als Hasenmama auftraten. Eine Erzählung über die Ereignisse zu Hause wäre ihm nicht über die Lippen gekommen.

Dieses Stop-Motion-Video wurde in der Therapie auf Wunsch des Kindes auch in der Kommunikation mit der Mutter genutzt, von der sich der Bub mehr Schutz in schwierigen Situationen wünschte. Beide waren verängstigt, da der Kindesvater trotz der bereits vor Jahren erfolgten Trennung der Eltern immer wieder im Lebensumfeld der beiden auftauchte und sie mit dem Auto verfolgte oder die Kindesmutter am Telefon belästigte und mit Entführung des Buben drohte.

Über das Video konnte die Mutter nach mehreren Eltern-Sitzungen schließlich aus ihrer Rolle als Hasenmama aussteigen und etwas mehr zur schützenden Bärenmama werden, die aktiv wurde und sich effizient Hilfe holte, um ihr Leben mit ihrem Sohn ungestört weiterführen zu können. Gemeinsam wurden in der Therapie in weiterer Folge Möglichkeiten erarbeitet, wie der Bub zuverlässige Freundschaften aufbauen konnte und nach dem Schulwechsel in eine weiterführende Schule nicht mehr als Mobbingopfer zur Verfügung stand. Da der Patient das Video auf seinem Handy gespeichert hatte, konnte er jederzeit die Szene ansehen und auch den Personen 
Abb. 3 Szene aus Stop-Motion-Video mit Krokodilvater, Bärenkind und Hasenmama

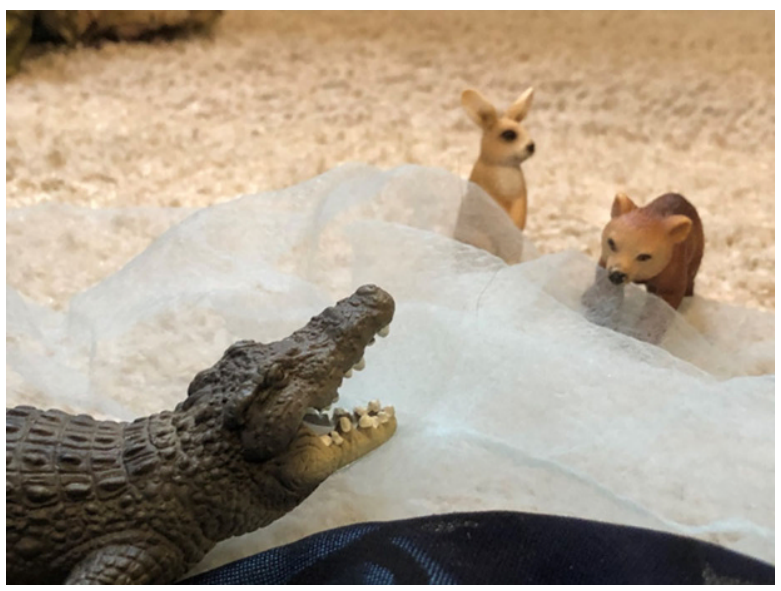

zeigen, denen er es zeigen wollte. Er setzte sich immer wieder mit seinen BärenTeilen und seinen Krokodil-Teilen auseinander (siehe Abb. 3).

Hier wurde das Intermediärobjekt „Stop-Motion-Video“ in seiner unterstützenden, vermittelnden und kreativen Funktion eingesetzt. Als Intraintermediärobjekt setzte es der junge Patient selbst ein, indem er es als Katalysator in der Kommunikation mit sich selbst verwendete und in weiterer Folge auch für die Kommunikation mit seiner Mutter und im Freundeskreis nützte.

Bei Stop-Motion-Videos ist es durch den schrittweisen Aufbau der Geschichte und der Spielszene für den/die PatientIn möglich, unzählige kleine Rollenwechsel zu vollziehen und das Tempo der Entfaltung der Szene dem eigenen innerseelischen Tempo anzupassen. Hier kommt es während des Filmens durch die wiederholten Standbilder immer wieder zum „Einfrieren“ der Szene und zum Gespräch mit dem/der TherapeutIn über die Entwicklung. Die entstehenden Gefühle der einzelnen MitspielerInnen bekommen Raum, sodass die Hilfs-Ich-Funktion und die verschiedenen Formen des Doppelns des/der TherapeutIn nutzbar werden, wenn dem/der PatientIn Ausdrucksmöglichkeiten fehlen. Auf diese Art und Weise kann, wie auch sonst im Psychodrama üblich, schrittweise Mentalisierung geübt werden. Diese Technik ist besonders für Menschen einsetzbar, die Schwierigkeiten haben, sich in andere einzufühlen, wie zum Beispiel Menschen mit der Diagnose einer Autismusspektrumsstörung oder schizoiden Persönlichkeiten, komplex traumatisierten Menschen, die zu Dissoziation oder dissozialem Verhalten neigen. Denn durch das kleinschrittige Vorgehen und die langsame Entwicklung der Szene kann über jede Sequenz gesprochen und auftretenden Gefühlen gemeinsam nachgegangen werden.

Ein Dialog mittels Rollentausch mit dem Computer, der Computermaus, dem Handy als Intermediärobjekt ist im Psychodrama mit Kindern und Jugendlichen möglich und wird zum Beispiel bei Mediensuchtthemen sinnvoll eingesetzt, allerdings fokussiere ich in diesem Artikel auf den Einsatz digitaler Medien als Bühne und als therapeutische Hilfsmittel oder Therapieunterstützer. 


\subsubsection{Rollenwechsel im Gruppensetting}

Der Rollenwechsel kann in der Gruppe per Videotelefonie oder -meeting in Form der Arbeit mit Symbolen auf der Tischbühne im Raum des/der TherapeutIn leicht durchgeführt werden, indem der/die TherapeutIn stellvertretend den Finger auf ein Symbol legt und den/die ProtagonistIn aus der entsprechenden Rolle heraus sprechen lässt.

Das unmittelbare und ganzheitliche Erleben der Arbeit auf der Tischbühne wird für PatientInnen allerdings dann intensiver, wenn sich die Tischbühne mit den Symbolen in deren Räumlichkeiten befindet. Sie wählen die Symbole unterstützt durch die Fragen des/der TherapeutIn in der eigenen Umgebung selbst aus, berühren danach auch noch selbst die Symbole und sprechen aus den entsprechenden Rollen heraus. Diese Form der Arbeit verlangt nach einer Absprache zwischen TherapeutIn und PatientIn und eventuell einigen Vorbereitungen vor der geplanten Sitzung (Altendorfer-Kling 2020, S. 378).

\subsection{Soziometrie}

Es hat sich bewährt, soziometrische Techniken wie das Soziogramm oder das Soziale Atom per Videomeeting auf einem Flipchart, als Power-Point-Präsentation oder White Board zu präsentieren, die PatientInnen ihre Position im Verhältnis zu den entsprechenden Kriterien beschreiben zu lassen und sie dann mittels kleiner Notizzettel oder Markierungen anzubringen. Auch die persönliche Position der PatientInnen nahe oder weiter weg vom Bildschirm kann im Rahmen soziometrischer Auswahlprozesse nutzbar gemacht werden, ebenso die Video-Tools „Handheben“, „Daumen hoch oder runter“, „Klatschen“. Zur Rollenwahl für ein Soziodrama durch die TeilnehmerInnen einer Gruppe ist es sehr nützlich, Post-Its bereit zu haben, auf die jedes Gruppenmitglied seine Wahl schreibt und diese Wahlen werden dann zugleich sichtbar gemacht.

Einen weiteren digitalen psychodramatischen Ansatz habe ich in der Vermittlung des „Roten Fadens der Zeit“ im Artikel „Psychodrama goes digital“ (AltendorferKling 2020, S. 378-379) bereits dargestellt. Hier wurde auf digitale Medien zurückgegriffen, um Treffen einer Selbsterfahrungsgruppe, die wegen des Lockdowns im Präsenzmodus nicht mehr weiterzuführen war, vorübergehend unterstützt durch digitale Medien zu ermöglichen. Es wurden Videokonferenz- und WhatsApp-Gruppen im Sinne der Blended Care in allen Phasen der Psychodrama-Sitzungen eingesetzt. Wir nützten vorrangig die vermittelnde Funktion des Therapieunterstützers ,Digitale Medien“. Der Einsatz der WhatsApp-Gruppe erwies sich als sehr hilfreich, um TeilnehmerInnen mit Internet-Verbindungsproblemen bestmöglich an der Gruppe teilhaben zu lassen. Es zeigte sich dadurch außerdem eine Stabilisierung der Gruppendynamik und Förderung der Gruppenkohäsion in den Zeiten zwischen den Gruppentreffen. 


\section{Analoger Einsatz digitaler Medien: Die imaginative Videokonferenztechnik}

Stellen wir das bisher Geschriebene auf den Kopf, dann wird eine weitere Einsatzmöglichkeit der digitalen Medien im Psychodrama sichtbar. Wir führen nun einen Bühnenwechsel von der digitalen auf die Bühne der Imagination (innere Bühne) durch. Die Bühne der Imagination besteht darin, die Videokonferenz im Face-toFace-Kontakt imaginativ mit Jugendlichen in Szene zu setzen, ähnlich der Bildschirmtechnik zur Traumakonfrontation von Frank Putnam (1989), Ulrich Sachsse, Lutz Ulrich Besser und Luise Reddemann (Reddemann, Hoffmann und Gast 2004, S. 135), beschrieben von Katharina Sternek (2014, S. 20-29). Die Jugendlichen sind inzwischen durch das Homeschooling schon sehr geübt im Umgang mit den Möglichkeiten der Videokonferenztools.

Bei dieser Form der Weiterentwicklung der Bildschirmtechnik bekommt der/die PatientIn die Rolle des Hosts einer imaginierten Videokonferenz angeboten und kann nun entweder andere Menschen oder seine Ich-Teile auf der inneren Bühne zu dieser Videokonferenz im monodramatischen Rollenspiel einladen und mit ihnen kommunizieren. Er oder sie wird sozusagen zum persönlichen Ausdruck ermächtigt und hat alle Möglichkeiten zur Wahl, die es bei einer Videokonferenz gibt. Das Mikrofon oder die Kamera der TeilnehmerInnen können ein-/ausgeschaltet werden, die Lautstärke ist variabel, es ist möglich, den Einen oder die Andere „stumm“ zu schalten oder ohne Video teilnehmen zu lassen. Auch ,unbeabsichtigte“ Gesprächsunterbrechungen oder Standbilder können gespielt werden. Diese Form des psychodramatischen Spiels wird sehr gern angenommen und ist beziehungsdiagnostisch sehr wertvoll. Die imaginierten TeilnehmerInnen können früher oder erst später aus dem Vorraum in den Besprechungsraum eingeladen werden, imaginierte Bildschirmfreigaben sind möglich.

Nebenbei kann via ,,privater Chatfunktion“ oder am imaginierten Handy mit einem Alter Ego oder Double beiseitegeschrieben werden. Im Theater wird auf der Bühne beiseitegesprochen (A-part-Sprechen), das heißt, die DialogpartnerInnen auf der Bühne bekommen es nicht mit, wohl aber das Publikum. In unserem Fall schreibt der/die ProtagonistIn in der Imagination beiseite, sodass es die imaginierten AntagonistInnen nicht mitbekommen, wohl aber das Double oder Alter Ego und der/die TherapeutIn.

Rollentausch bzw. Rollenwechsel mit eingeladenen TeilnehmerInnen an der Videokonferenz ist szenisch möglich, indem aus der Rolle eines der Ego States oder der Teile nach Aichinger (2007, S. 67-82) mit dem Selbst (Host der Videokonferenz) gesprochen wird.

Mit Jugendlichen ab dem Alter von zehn Jahren habe ich damit sehr gute Erfahrungen gemacht, indem ich ihnen ein Videomeeting als Spielszene angeboten und sie danach gefragt habe, um welches Thema oder welche Fragestellung es gehen sollte und welche Personen sie einlüden, wenn sie Host eines Videomeetings wären. Als Host genossen sie dann ihre Macht in der Regieführung. Sie wurden sehr kreativ und konnten auf dieser Bühne unter anderem gefahrlos ihre boshaften Seiten ausspielen und Rachefantasien inszenieren. Die imaginative Videotechnik ermöglicht außerdem einen Einblick in das Soziale Atom der Jugendlichen. 
In der Krisenintervention ist das „Komplettieren der Szene“ methodenübergreifend für alle Beteiligten ein Grundkonzept. Im Psychodrama ist das szenische Denken und genaue Erfragen der Lage selbstverständlich. Bei länger zurückliegenden Traumatisierungen kann die Anamnese nur in kleinen Schritten erfolgen (Pruckner 2014, S. 171). Dies kann durch die imaginative Videokonferenztechnik im geschützten Rahmen sehr einfühlsam und step by step über mehrere Sitzungen erfolgen. Die Traumaanamnese und die Reaktionen der Umwelt oder des Sozialen Atoms können mithilfe der imaginativen Videokonferenztechnik exploriert werden. Außer den Faktoren Alter des/der PatientIn, Art und Dauer des Traumas, sind die pathologisierenden oder protektiven Reaktionen des engen und weiteren Umfelds unmittelbar beim traumatischen Ereignis, aber auch später sehr entscheidend bei der Traumabewältigung. Traumafolgen sind intra- und interpersonal (Pruckner 2014, S. 172).

In der Rolle des Hosts ist der/die PatientIn in einer machtvollen Rolle und kann die Szene aus verschiedenen Rollen heraus bearbeiten, sich mit AntagonistInnen auseinandersetzen, ohne in die Rolle des Opfers zu rutschen. Der/die TherapeutIn arbeitet mit der Interviewtechnik und begleitet den/die PatientIn durch das szenische Spiel.

Traumaintegration erfolgt bei weitem nicht ausschließlich über ,,aufdeckende Bearbeitung“. Daher wird auch mithilfe digitaler Medien traumasensibel gearbeitet. Die Videokonferenztechnik ermöglicht dem Host viele Möglichkeiten, Dinge nur anzudeuten oder im Schutz eines Symbols zu verpacken. Über den virtuellen Warteraum kann zum Beispiel die benötigte Distanz zu bestimmten TeilnehmerInnen des imaginierten Meetings gewahrt werden. Die Funktion des vituellen Hintergrunds ermöglicht es, Dinge zu verstecken. TeilnehmerInnen können stummgeschaltet oder ihnen eine Teilnahme am Meeting verwehrt werden.

Ähnlich der Tresorübung von Luise Reddemann (2001) können Ereignisse/ Dateien in den unendlichen Tiefen des Computers an mehrfach abgesicherten Orten versenkt werden.

Als digitale Bühnen für vermittelnde Intermediärobjekte können prinzipiell alle MMPORGs (Massively Multi-Player-Online-Role-Games) dienen, auch Spiele wie Minecraft, World of Warcraft oder z. B. The Legend of Zelda.

Fallbeispiel: Ein jugendlicher Patient entwickelte in seiner Psychodramatherapie gemeinsam mit mir eine Wunschfantasie für seinen zukünftigen Lebensentwurf. Auf die blanke Frage, wie er sich seine Zukunft vorstelle, was er in zehn oder fünfzehn Jahren einmal machen wollte, kam als Antwort nur unschlüssiges Schulterzucken.

Seine Erzählungen über sein Spielverhalten und das der Mitspieler inspirierte uns dann zu einer individuellen Umgestaltung des Spieles und zum Neuschreiben der Legend of Zelda zu einer persönlichen Legende des jungen Mannes. Wir entwickelten gemeinsam einige Szenen zunächst im Sinne eines Drehbuchs für das Spiel. Dann spielten wir im Einzelsetting während mehrerer Therapiesitzungen die Schlüsselszenen, manchmal sehr kurze Sequenzen im (an Bäumen, Felsen und Gebüsch reichen) Garten der Psychotherapiepraxis nach. Diese wurden auf Wunsch des Patienten auf dessen Handy als Kurzvideos, Slow-Motion-Videos oder Standbilder 
von mir aufgenommen. Er selbst kreierte mithilfe einer App einen kurzen Film über seine Zukunftsfantasie im Reich der Zelda.

Hier entwickelte sich das Intermediärobjekt im weiteren Therapieverlauf zu einem Intra-Intermediärobjekt mit vermittelnder, unterstützender und kreativer Funktion. Viele Jugendliche lieben es, über ihre Quests zu erzählen. Das ermöglicht, dass man die narrativen Spielelemente der Computerspiele in die Therapie hereinholen kann, indem man zum Beispiel die Frage stellt: „Wie würdest du Quests in der Rolle eines/r SpieleentwicklerIn nutzen?“"

\section{Diskussion}

Die Nutzung der digitalen Medien im Rahmen der akuten Krisenintervention und Traumatherapie bietet viele Möglichkeiten und Entwicklungspotentiale. Ein Beispiel ist laut Labatzki (2020) das bekannte, aus den 1980er-Jahren stammende PC-Spiel „Tetris“, das sich in einer Studie der University of Oxford als wirksam in der Traumatherapie erwiesen hat und flashbackartige Erinnerungen reduzieren hilft, ähnlich wie die Formen der bilateralen Stimulierung von Trauma-Netzwerken in der EMDRBehandlung von Menschen mit Posttraumatischen Belastungsstörungen.

In schweren Krisensituationen, wie bei akuter Suizidalität, ist meist eine direkte menschliche Begegnung einem Gespräch per Bildschirm oder Tastatur vorzuziehen. Die Einhaltung datenschutzrechtlicher Bestimmungen ist herausfordernd. Daher bietet die Internetrichtlinie für Psychotherapeutinnen und Psychotherapeuten in Österreich hier nach Hintenberger (2012) eine sehr fundierte und regelmäßig aktualisierte Orientierung.

In quantitativer oder qualitativer Hinsicht „falscher“ Medienkonsum ist durchaus schädlich für die kognitive, psychosoziale und ggf. körperliche Entwicklung von Kindern und Jugendlichen. Personale, positiv besetzte Beziehungen zu einfühlsamen Bezugspersonen können nicht durch virtuelle Beziehungen ersetzt werden: Unter Umständen ist bei Internetsucht der Verdacht auf eine unsichere Bindung gegeben (vgl. Eichenberg 2014). Orientierende und strukturierte Lebenskontexte (Grenzen, Werte und Regeln) auf der Basis einer sicheren Bindung (gesprächsbereite, aneinander interessierte Eltern und Kinder) schützen Kinder und Jugendliche am besten vor Mediensuchtgefahren und vor Cybergrooming.

In Bezug auf die oben beschriebenen Beispiele ist mir wichtig festzuhalten, dass ich nicht mittels digitaler Medien den persönlichen Kontakt ersetze. Ich sehe die digitalen Medien in der Psychodramatherapie dort als wertvolle Ergänzung und Möglichkeit zur Kommunikation, wo anders keine Begegnung möglich wäre (Menschen aus entlegenen Regionen).

\section{Fazit}

„Das Spiel ist der Königsweg der Kinder.“ (Pruckner 2001) - Das Videospiel ist der Königsweg der Jugendlichen. 
In der Therapie von Kindern und Jugendlichen ist ein wichtiger Grundsatz, das Kind oder den jugendlichen Menschen dort abzuholen, wo er/sie steht und ihm/ihr Entwicklung in seinem eigenen Tempo zu ermöglichen. Ganz nach dem afrikanischen Sprichwort: Gras wächst nicht schneller, wenn man daran zieht.

Daher ist es eine Chance für uns TherapeutInnen, wenn wir uns mit den aktuellen Ausdrucksformen junger Menschen auseinandersetzen, um ihnen eine entsprechende Begegnungsmöglichkeit anzubieten. Snapchat, TikTok und Instagram oder wie sie alle heißen sind schnelllebige Foren des Selbstausdrucks und möchten durchaus auch imaginativ in die Therapie hereingeholt werden. Wir können die Kreativität der Videospiele und Social-Media-Foren für die psychodramatische Arbeit mit Kindern und Jugendlichen nutzbar machen.

Laut Charles Darwin ist es nicht die stärkste Spezies, die überlebt, auch nicht die intelligenteste, es ist diejenige, die sich am ehesten dem Wandel anpassen kann (Wirsching 2017). Kinder und Jugendliche können sich noch an vieles anpassen und lernen gut damit umzugehen und zu leben.

Nach allem, was ich von J. L. Moreno gelesen und erfahren habe, schätze ich ihn als einen Menschen ein, der sich die Offenheit für Veränderung, wie wir sie von Kindern und Jugendlichen kennen, bewahrt hat. Folgen wir ihm auf der „Road to the Future" (Video 2020).

Es ist wünschenswert, grundsätzlich darüber nachzudenken, inwiefern die Nutzung der digitalen Bühne auch nach der Covid-19-Pandemie eine Bereicherung für das (psychodrama-) therapeutische Arbeiten darstellt und beispielsweise zur Erreichung neuer Zielgruppen beitragen könnte.

\section{Literatur}

Aichinger, A. (2007). Warum der kleine Löwe immer bissiger wird. Frühe Intervention bei einem aggressiven Kind. ZPS, 6, 67-82. https://doi.org/10.1007/s11620-007-0006-1.

Aichinger, A. (2012). Einzel- und Familientherapie mit Kindern. Kinderpsychodrama, Bd. 3 (S. 175-176). Wiesbaden: Springer VS.

Altendorfer-Kling, U. (2020). Psychodrama goes digital! Z Psychodrama Soziom, 19, 375-387. https://doi. org/10.1007/s11620-020-00554-0.

(2017). Glossar. In G. Biegler-Vitek \& M. Wicher (Hrsg.), Theorie und Praxis der Psychodrama-Psychotherapie. In der Anwendung mit Eltern, Kindern und Jugendlichen (S. 272). Wien: Facultas.

Blahacek, A. (2020). Psychodramatische Gruppentherapie per Skype - geht das überhaupt?! Z Psychodrama Soziom, 19, 365-374. https://doi.org/10.1007/s11620-020-00553-1.

Eichenberg, C. (2014). Verhaltenssüchte: Internetsucht geht mit unsicherer Bindung einher. Deutsches Ärzteblatt, 12(6), 269-271.

Hintenberger, G. (2012). Die Internetrichtlinie für Psychotherapeutinnen und Psychotherapeuten in Österreich - Eine kritische Würdigung. Fachzeitschrift für Onlineberatung und computervermittelte Kommunikation, 1,3 .

Labatzki, U. (2020). Digitale Infekte - Digitale Heilung, Krankheit und Therapie im Zeitalter der Digitalisierung. In K. Heiland (Hrsg.), Prinzip Infektion, Atmosphärische Übertragung in Gesellschaft, Kunst und Psychoanalyse. Gießen: Psychosozialverlag.

Moreno, J.L. Psychodrama in Action: „A Road to the Future“. https://www.youtube.com/watch? $\mathrm{v}=$ Bdu8jTs-fnA. Zugegriffen: 4. Nov. 2020.

Pruckner, H. (2001). Das Spiel ist der Königsweg der Kinder. München: inScenario.

Pruckner, H. (2002). Du Sollst nicht fragen, das Kind will nicht reden. ZPS, 1, 147-175. https://doi.org/ 10.1007/s11620-002-0017-x.

Pruckner, H. (2004). Arbeit mit intermediären und intraintermediären Objekten. In J. Fürst, K. Ottomeyer \& H. Pruckner (Hrsg.), Psychodrama-Therapie. Ein Handbuch (S. 266-278). Wien: Facultas. 
Pruckner, H. (2014). Psychodramatische Arbeit mit traumatisierten Kindern. In G. Biegler-Vitek \& M. Wicher (Hrsg.), Psychodrama-Psychotherapie mit Kindern und Jugendlichen. Ein Handbuch (S. 168-187). Wien: Facultas.

Reddemann, L. (2001). Imagination als heilsame Kraft: Ressourcen und Mitgefühl in der Behandlung von Traumafolgen. Stuttgart: Klett-Cotta.

Reddemann, L., Hoffmann, A., \& Gast, U. (Hrsg.). (2004). Psychotherapie der dissoziativen Störungen (Lindauer Psychotherapie Module). Stuttgart, New York: Thieme.

Rojas-Bermúdes, J. G. (1985). Puppets and psychodrama (2. Aufl.). Buenos Aires: Editorial Celsius.

Sachsse, U. (2004). Traumazentrierte Psychotherapie. Stuttgart: Schattauer.

Schulte-Markwort, M. (2020). Seelenleben: Einblicke in die jugendliche Psyche. Hamburg: Carlsen.

Sternek, K. (2014). Über den Einsatz und die Wirkungsweise von „Bildschirm-Techniken“. Phänomenal, 6(1), 20-29.

Wirsching, K. T. (2017). Motivation und Zielsetzung. In Familieneinfluss und Unternehmenserfolg. Wiesbaden: Gabler. https://doi.org/10.1007/978-3-658-18955-6_1.

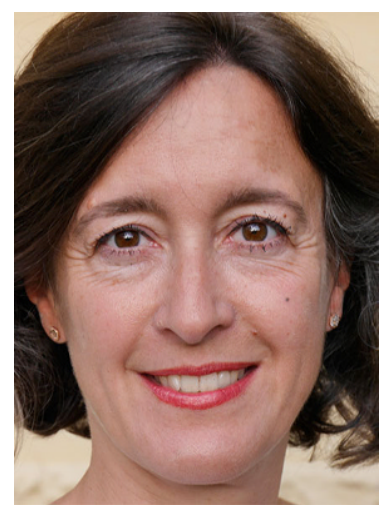

Dr. Ulrike Altendorfer-Kling 1972, Dr. med. univ., Fachärztin für Kinder- und Jugendpsychiatrie und Psychotherapeutische Medizin, Ärztliche Leiterin der Kinder-Jugend-Seelenhilfe Salzburg, Lehrende an der Paracelsus Medizinischen Privatuniversität Salzburg, Lehrtherapeutin mit partieller Lehrbefugnis für Psychodrama, Soziometrie und Rollenspiel (ÖAGG), Lehrbeauftragte für Psychosoziale, Psychosomatische und Psychotherapeutische Medizin der Ö̈̈K, Gründerin der AG Kids' Bridge, Mitglied der ÖGKJP, Psychodrama-Psychotherapeutin in freier Praxis mit Weiterbildung in Säuglings-, Kinder- und Jugendlichenpsychotherapie 